\title{
Construction Integrity and Professionalism of Education Personnel
}

\author{
$1^{\text {st }}$ Warsono \\ Department of Pancasila and \\ Citizenship Education, Faculty of \\ Social Sciences and Law \\ Universitas Negeri Surabaya \\ Surabaya, Indonesia \\ Email: warsono@unesa.ac.id
}

\author{
$2^{\text {nd }}$ Sarmini \\ Department of Pancasila and \\ Citizenship Education, Faculty of \\ Social Sciences and Law \\ Universitas Negeri Surabaya \\ Surabaya, Indonesia \\ Email: sarmini@unesa.ac.id
}

\author{
$3^{\text {rd }}$ Ulin Nadiroh \\ Department of Social Studies \\ Universitas Negeri Surabaya \\ Surabaya, Indonesia \\ Email: ulinnadiroh099@gmail.com
}

\begin{abstract}
Education personnel are Human Resources who have an important role to support the performance of academics, who are obliged to provide academic services. In carrying out its duties, can not be separated from the existence of interdependence and even the relationship between one unit to another unit. This paper aims to find out the level of understanding of integrity and professionalism level of education personnel. The method in this study is exploratory study. The subjects taken were the head of sub-division at Universitas Negeri Surabaya (UNESA). Data collection techniques used are kusioner. The result of this research is that the integrity and professionalism of the education personnel at UNESA has been functionally good, but structurally there still needs to be improvement. So it is necessary to have various training courses to support the quality of the performance of all parties and it is considered important.
\end{abstract}

Keywords - integrity, professionalism, education personnel

\section{INTRODUCTION}

In the current global era, education is required to have an important role, namely having to prepare quality human beings and be able to compete in accordance with the advancement of science and technology [1] or humans who have mental readiness and at the same time readiness of skill abilities and the world of education is able to prepare competent humans and creativity [2].

The quality of education is one of the national problems that often changes in all fields [3], [4]. Education and learning are required to account for their duties, which are required to carry out effective, efficient learning and follow the direction of the times [5]-[7]. The success factor of the education sector is not only from the educator, but the educational staff also determines the quality of education.

Educational personnel are the personnel who are tasked with planning and implementing administration, management, development, supervision, and technical services to support the educational process in educational units [8]. Another understanding of education personnel is human resources tasked with carrying out administration, management, development, supervision, and technical services to support the delivery of educational processes in education or research units [8]. Education personnel include school principals, education unit supervisors, administrative staff, library staff, laboratory personnel, technicians, study group managers, study tutors, and cleaning staff [9].
Education personnel are one of the factors that influence the success of the quality of education quality [5]. In order to achieve success in education, it is necessary to increase integrity and professionalism in education personnel [10]. The quality of human resources (HR) is highly dependent on intelligence, honesty, skills, creativity, and professionalism in science and management [11]. The success of national development is determined by the quality of human resources. Reliable human resources (HR) are a strong foundation in dealing with changing times [12] and excellent performance achievement in an organization [13]. Education personnel are an important element in the effectiveness of education.

Educators and education personnel in the education process play a strategic role, especially in efforts to shape the character of the nation through the development of desirable personalities and values [14]. To understand the concept of management of educators and educators, we must first understand the meaning of management and educators and education [15].

The quality of education is in line with the demands of development and change [3], [16]. A change requires the role of a renewal agent (the agent of change) in generating ideas for reform and managing change. Some internal change agent figures in educational institutions are human resources, namely educators and education personnel who are allegedly loaded with various problems [17], including professional qualifications, coaching and development, and their performance that requires intense attention, direction and intensive guidance and sustainable [18], so that it is truly capable of carrying out all of its duties, functions and responsibilities professionally, in line with the required standards of educators and education personnel [15], [19]. In general education, staff has diverse academic qualifications and competencies. This condition has an impact on the quality of the services it provides as well as on the level of integrity [20], the speed of adapting to technological developments [4] to adapting to leadership performance. Therefore the level of integrity and professionalism level of education personnel is considered important to be improved [21]. Thus the need for integrity and professionalism in work [22], [23].

Integrity is one of the most important attributes a leader must have [24]. Integrity is a concept related to consistency in actions, values, methods, measures, principles, 
expectations and things that are produced [25]. People with integrity means having an honest and strong character [24].

In addition to integrity, education staff must also have professionalism at work [26]. Professionalism, in this case, refers to the degree of one's appearance as a professional or the appearance of a job is considered a profession. There is a high, medium and low professionalism. The high and low recognition of professionalism is very dependent on the expertise and level of education that he takes and refers to the attitude [27]. Professionalism is the individual's responsibility to behave better than merely complying with existing laws and community regulations [28].

There are several criteria to make a person become a professional [29], one must also comply with existing standards and not take sides with a client. And must be responsible for the reports presented. According to general understanding, a person is said to be professional if it meets three criteria, namely having the expertise to carry out tasks in accordance with their fields, carrying out a task or profession by setting standard standards in the relevant profession, carrying out his professional duties by complying with established professional ethics [20].

The role of education personnel is to assist in managing educational institutions, help plan an educational design, help facilitate educational activities, help secure the educational environment, help create a beautiful and comfortable atmosphere of education [30]. Whereas the education staff function is to guarantee the continuity of an education system, monitor a system in educational institutions, provide facilities to educators and students in carrying out an educational activity, provide comfort and safety in the educational environment, and regulate an educational process in an institution [31].

Indeed, integrity and professionalism must be owned specifically by education personnel. Therefore, this paper discusses the construction of a level of understanding the integrity and professionalism level of education personnel at Universitas Negeri Surabaya towards leading and competitive universities.

\section{RESEARCH METHOD}

This research is an exploratory study. Data collection techniques used is questionnaire. Subjects respond with five categories of terms using the Likert Scale, which is: strongly disagree (1), disagree (2), agree (3), strongly agree (4), and strongly agree (5) with an answer score. A questionnaire regarding construction of understanding level of integrity and professionalism level of education personnel. The subject in this paper is the Head of Sub Division of 24 people at UNESA.

\section{RESULT AND DISCUSSION}

Educational staff or referred to as administrative staff in universities have the task of supporting various academic tasks and providing services in the field of administration. In general, administrative staff has diverse academic qualifications and competencies. This condition has an impact on the quality of the services it provides as well as on the level of integrity, professionalism, and the speed of adapting to technological developments to adapting to the leadership performance. Therefore, the level of integrity and professionalism level of education personnel is considered important to be improved. The recapitulation of understanding integrity and professionalism can be seen in Table 1.

TABLE. 1. INTEGRITY UNDERSTANDING RECAPITULATION AND PROFESSIONALITY OF THE HEAD OF SUB PART OF THE UNIVERSITAS NEGERI SURABAYA.

\begin{tabular}{|c|c|c|c|c|c|c|c|}
\hline \multirow[b]{2}{*}{ No. } & \multirow[b]{2}{*}{ Statement } & \multicolumn{5}{|c|}{ Scale (\%) } & \multirow{2}{*}{$\begin{array}{c}\text { Average } \\
(\%)\end{array}$} \\
\hline & & 5 & 4 & 3 & 2 & 1 & \\
\hline 1. & $\begin{array}{l}\text { An employee must have a } \\
\text { concept that points to } \\
\text { consistency between actions } \\
\text { and values and principles }\end{array}$ & $\begin{array}{c}(25) \\
27,5 \%\end{array}$ & $\begin{array}{c}(36) \\
39,6 \%\end{array}$ & $\begin{array}{l}(30) \\
33 \%\end{array}$ & $\begin{array}{l}(0) \\
0 \%\end{array}$ & $\begin{array}{l}(0) \\
0 \%\end{array}$ & $75,8 \%$ \\
\hline 2. & $\begin{array}{l}\text { A person must have } \\
\text { integrity, because integrity } \\
\text { shows honesty and truth of } \\
\text { one's actions }\end{array}$ & $\begin{array}{c}(40) \\
41,7 \%\end{array}$ & $\begin{array}{c}(32) \\
33,3 \%\end{array}$ & $\begin{array}{c}(24) \\
25,0 \%\end{array}$ & $\begin{array}{l}(0) \\
0 \%\end{array}$ & $\begin{array}{l}(0) \\
0 \%\end{array}$ & $80,0 \%$ \\
\hline 3. & $\begin{array}{l}\text { Every employee must have a } \\
\text { promise to themselves or } \\
\text { others reflected in the actions } \\
\text { of a person according to their } \\
\text { duties and functions } \\
\text { according to their position }\end{array}$ & $\begin{array}{c}(10) \\
11,6 \%\end{array}$ & $\begin{array}{c}(40) \\
46,5 \%\end{array}$ & $\begin{array}{c}(36) \\
41,9 \%\end{array}$ & $\begin{array}{l}(0) \\
0 \%\end{array}$ & $\begin{array}{l}(0) \\
0 \%\end{array}$ & $71,7 \%$ \\
\hline 4. & $\begin{array}{l}\text { Every employee does not } \\
\text { have to have a promise to } \\
\text { themselves or their leaders, } \\
\text { which is important to carry } \\
\text { out their duties well }\end{array}$ & $\begin{array}{l}(0) \\
0 \%\end{array}$ & $\begin{array}{l}(0) \\
0 \%\end{array}$ & $\begin{array}{c}(36) \\
40,0 \%\end{array}$ & $\begin{array}{c}(24) \\
26,7 \%\end{array}$ & $\begin{array}{c}(30) \\
33,3 \%\end{array}$ & $75,0 \%$ \\
\hline 5. & $\begin{array}{l}\text { Even though the leader is not } \\
\text { in the place, he will carry out } \\
\text { the duties in accordance with } \\
\text { the main tasks that he has }\end{array}$ & $\begin{array}{c}(75) \\
71,4 \%\end{array}$ & $\begin{array}{c}(12) \\
11,4 \%\end{array}$ & $\begin{array}{c}(18) \\
17,1 \%\end{array}$ & $\begin{array}{l}(0) \\
0 \%\end{array}$ & $\begin{array}{l}(0) \\
0 \%\end{array}$ & $87,5 \%$ \\
\hline 6. & $\begin{array}{l}\text { In carrying out the tasks } \\
\text { awaiting instructions from } \\
\text { superiors to be in accordance } \\
\text { with the wishes of the } \\
\text { leadership }\end{array}$ & $\begin{array}{c}(40) \\
40,8 \%\end{array}$ & $\begin{array}{c}(40) \\
40,8 \%\end{array}$ & $\begin{array}{c}(18) \\
18,4 \%\end{array}$ & $\begin{array}{l}(0) \\
0 \%\end{array}$ & $\begin{array}{l}(0) \\
0 \%\end{array}$ & $81,7 \%$ \\
\hline 7. & $\begin{array}{l}\text { Employees must keep all the } \\
\text { promises that have been } \\
\text { promised to their superiors }\end{array}$ & $\begin{array}{c}(85) \\
78,0 \%\end{array}$ & $\begin{array}{c}(12) \\
11,0 \%\end{array}$ & $\begin{array}{l}(12) \\
11 \%\end{array}$ & $\begin{array}{l}(0) \\
0 \%\end{array}$ & $\begin{array}{l}(0) \\
0 \%\end{array}$ & $90,8 \%$ \\
\hline 8. & $\begin{array}{l}\text { Even though you are at risk } \\
\text { of being sanctioned, you will } \\
\text { keep all the promises that } \\
\text { have been promised to your } \\
\text { boss }\end{array}$ & $\begin{array}{c}(60) \\
56,6 \%\end{array}$ & $\begin{array}{c}(40) \\
37,7 \%\end{array}$ & $\begin{array}{c}(6) \\
5,7 \%\end{array}$ & $\begin{array}{l}(0) \\
0 \%\end{array}$ & $\begin{array}{l}(0) \\
0 \%\end{array}$ & $88,3 \%$ \\
\hline 9. & $\begin{array}{l}\text { As an employee will say in } \\
\text { accordance with what is } \\
\text { seen, heard and felt about } \\
\text { various things that are } \\
\text { around the working } \\
\text { environment }\end{array}$ & $\begin{array}{c}(75) \\
70,1 \%\end{array}$ & $\begin{array}{c}(20) \\
18,7 \%\end{array}$ & $\begin{array}{c}(12) \\
11,2 \%\end{array}$ & $\begin{array}{l}(0) \\
0 \%\end{array}$ & $\begin{array}{l}(0) \\
0 \%\end{array}$ & $89,2 \%$ \\
\hline 10. & $\begin{array}{l}\text { Employees must maintain } \\
\text { harmony in the surrounding } \\
\text { environment, so there is no } \\
\text { need to say everything that is } \\
\text { happening around us, to the } \\
\text { leadership }\end{array}$ & $\begin{array}{l}(100) \\
86,2 \%\end{array}$ & $\begin{array}{c}(16) \\
13,8 \%\end{array}$ & $\begin{array}{l}(0) \\
0 \%\end{array}$ & $\begin{array}{l}(0) \\
0 \%\end{array}$ & $\begin{array}{l}(0) \\
0 \%\end{array}$ & $96,7 \%$ \\
\hline 11. & $\begin{array}{l}\text { In working must have high } \\
\text { solidarity, so there is no need } \\
\text { to say the mistakes of the } \\
\text { children or friends to the } \\
\text { leadership }\end{array}$ & $\begin{array}{c}(90) \\
80,4 \%\end{array}$ & $\begin{array}{c}(16) \\
14,3 \%\end{array}$ & $\begin{array}{c}(6) \\
5,4 \%\end{array}$ & $\begin{array}{l}(0) \\
0 \%\end{array}$ & $\begin{array}{l}(0) \\
0 \%\end{array}$ & $93,3 \%$ \\
\hline 12. & $\begin{array}{l}\text { An employee must have } \\
\text { kestiaan to his institution, by } \\
\text { working hard }\end{array}$ & $\begin{array}{c}(115) \\
96,6 \%\end{array}$ & $\begin{array}{c}(4) \\
3,4 \%\end{array}$ & $\begin{array}{l}(0) \\
0 \%\end{array}$ & $\begin{array}{l}(0) \\
0 \%\end{array}$ & $\begin{array}{l}(0) \\
0 \%\end{array}$ & $99,2 \%$ \\
\hline 13. & $\begin{array}{l}\text { Who is the boss will } \\
\text { influence the performance } \\
\text { donated to the institution }\end{array}$ & $\begin{array}{c}(95) \\
84,1 \%\end{array}$ & $\begin{array}{c}(12) \\
10,6 \%\end{array}$ & $\begin{array}{c}(6) \\
5,3 \%\end{array}$ & $\begin{array}{l}(0) \\
0 \%\end{array}$ & $\begin{array}{l}(0) \\
0 \%\end{array}$ & $94,2 \%$ \\
\hline 14. & $\begin{array}{l}\text { Every day will carry out the } \\
\text { task without any sense of } \\
\text { hesitation and confusion }\end{array}$ & $\begin{array}{c}(12) \\
57,7 \%\end{array}$ & $\begin{array}{c}(8) \\
30,8 \%\end{array}$ & $\begin{array}{c}(4) \\
11,5 \%\end{array}$ & $\begin{array}{l}(0) \\
0 \%\end{array}$ & $\begin{array}{l}(0) \\
0 \%\end{array}$ & $86,7 \%$ \\
\hline 15. & $\begin{array}{l}\text { Reprimand friends or } \\
\text { subordinates, with great care } \\
\text { and based on strong facts }\end{array}$ & $\begin{array}{c}(12) \\
55,6 \%\end{array}$ & $\begin{array}{l}(12) \\
44,4 \%\end{array}$ & $\begin{array}{l}(0) \\
0 \%\end{array}$ & $\begin{array}{l}(0) \\
0 \%\end{array}$ & $\begin{array}{l}(0) \\
0 \%\end{array}$ & $90,0 \%$ \\
\hline 16. & $\begin{array}{l}\text { Comply with applicable } \\
\text { regulations, without seeing }\end{array}$ & $\begin{array}{c}(20) \\
86,2 \%\end{array}$ & $\begin{array}{c}(4) \\
13,8 \%\end{array}$ & $\begin{array}{l}(0) \\
0 \%\end{array}$ & $\begin{array}{l}(0) \\
0 \%\end{array}$ & $\begin{array}{l}(0) \\
0 \%\end{array}$ & $96,7 \%$ \\
\hline
\end{tabular}




\begin{tabular}{|c|c|c|c|c|c|c|c|}
\hline & the presence of superiors & & & & & & \\
\hline 17. & $\begin{array}{l}\text { Believing that self-discipline } \\
\text { is the first step in building a } \\
\text { career }\end{array}$ & $\left|\begin{array}{c}(22) \\
93,2 \%\end{array}\right|$ & $\begin{array}{c}(2) \\
6,8 \%\end{array}$ & $\begin{array}{l}(0) \\
0 \%\end{array}$ & $\begin{array}{l}(0) \\
0 \%\end{array}$ & $\begin{array}{l}(0) \\
0 \%\end{array}$ & $98,3 \%$ \\
\hline 18. & $\begin{array}{l}\text { The closeness of the } \\
\text { relationship with the } \\
\text { leadership becomes an } \\
\text { important dimension, rather } \\
\text { than a disciplined attitude }\end{array}$ & $\begin{array}{l}(0) \\
0 \%\end{array}$ & $\begin{array}{l}(0) \\
0 \%\end{array}$ & $\begin{array}{l}(0) \\
0 \%\end{array}$ & $\begin{array}{l}(4) \\
13,8 \%\end{array}$ & $\begin{array}{c}(20) \\
86,2 \%\end{array}$ & $96,7 \%$ \\
\hline 19. & $\begin{array}{l}\text { What is done in building self } \\
\text { quality is by working hard }\end{array}$ & \begin{tabular}{|c|}
$(20)$ \\
$86,2 \%$ \\
\end{tabular} & \begin{tabular}{|c|}
$(4)$ \\
$13,8 \%$ \\
\end{tabular} & $\begin{array}{l}(0) \\
0 \% \\
\end{array}$ & $\begin{array}{l}(0) \\
0 \%\end{array}$ & $\begin{array}{l}(0) \\
0 \%\end{array}$ & $96,7 \%$ \\
\hline 20. & $\begin{array}{l}\text { Angry to a friend who } \\
\text { determines my quality is } \\
\text { bad. }\end{array}$ & $\begin{array}{l}(0) \\
0 \%\end{array}$ & $\begin{array}{l}(0) \\
0 \%\end{array}$ & $\begin{array}{l}(0) \\
0 \%\end{array}$ & $\begin{array}{l}(12) \\
44,4 \%\end{array}$ & $\begin{array}{c}(12) \\
55,6 \%\end{array}$ & $90,0 \%$ \\
\hline & Professionalism & & & & & & \\
\hline 21. & $\begin{array}{l}\text { A Professional employee } \\
\text { will carry out tasks and work } \\
\text { supported by abilities, } \\
\text { knowledge, skills, ability to } \\
\text { do and experience }\end{array}$ & $\left|\begin{array}{l}(100) \\
86,2 \%\end{array}\right|$ & $\begin{array}{c}(16) \\
13,8 \%\end{array}$ & $\begin{array}{l}(0) \\
0 \%\end{array}$ & $\begin{array}{l}(0) \\
0 \%\end{array}$ & $\begin{array}{l}(0) \\
0 \%\end{array}$ & $96,7 \%$ \\
\hline 22. & $\begin{array}{l}\text { Adjust the capabilities } \\
\text { possessed by the demands of } \\
\text { the main tasks that are } \\
\text { owned }\end{array}$ & $\begin{array}{c}(30) \\
31,3 \%\end{array}$ & $\begin{array}{c}(48) \\
50,0 \%\end{array}$ & $\begin{array}{c}(18) \\
18,8 \%\end{array}$ & $\begin{array}{l}(0) \\
0 \%\end{array}$ & $\begin{array}{l}(0) \\
0 \%\end{array}$ & $80,0 \%$ \\
\hline 23. & $\begin{array}{l}\text { Shame if you do not have the } \\
\text { ability in accordance with } \\
\text { the tasks and functions they } \\
\text { have }\end{array}$ & $\mid \begin{array}{l}(110) \\
93,2 \%\end{array}$ & $\begin{array}{c}(8) \\
6,8 \%\end{array}$ & $\begin{array}{l}(0) \\
0 \%\end{array}$ & $\begin{array}{l}(0) \\
0 \%\end{array}$ & $\begin{array}{l}(0) \\
0 \%\end{array}$ & $98,3 \%$ \\
\hline 24. & $\begin{array}{l}\text { To produce maximum work } \\
\text { products, must synergize } \\
\text { with other friends }\end{array}$ & $\begin{array}{c}(90) \\
80,4 \%\end{array}$ & $\begin{array}{c}(19) \\
14,3 \%\end{array}$ & $\begin{array}{c}(6) \\
5,4 \%\end{array}$ & $\begin{array}{l}(0) \\
0 \%\end{array}$ & $\begin{array}{l}(0) \\
0 \%\end{array}$ & $93,3 \%$ \\
\hline 25. & $\begin{array}{l}\text { In carrying out the task there } \\
\text { is no need to ask help from } \\
\text { other friends, for fear of } \\
\text { disturbing }\end{array}$ & $\begin{array}{c}(20) \\
23,3 \%\end{array}$ & $\begin{array}{c}(32) \\
37,2 \%\end{array}$ & $\begin{array}{c}(30) \\
34,9 \%\end{array}$ & $\begin{array}{c}(4) \\
4,7 \%\end{array}$ & $\begin{array}{l}(0) \\
0 \%\end{array}$ & $71,7 \%$ \\
\hline 26. & $\begin{array}{l}\text { Build cohesiveness with } \\
\text { friends, because } \\
\text { cohesiveness is the basic } \\
\text { foundation for success }\end{array}$ & $\begin{array}{c}(80) \\
74,1 \%\end{array}$ & $\begin{array}{c}(16) \\
14,8 \%\end{array}$ & $\begin{array}{c}(12) \\
11,1 \%\end{array}$ & $\begin{array}{l}(0) \\
0 \%\end{array}$ & $\begin{array}{l}(0) \\
0 \%\end{array}$ & $90,0 \%$ \\
\hline 27. & $\begin{array}{l}\text { Every time there is a division } \\
\text { of tasks from the boss, } \\
\text { friends will carry out tasks } \\
\text { that are properly charged }\end{array}$ & $\begin{array}{c}(90) \\
80,4 \%\end{array}$ & $\begin{array}{c}(16) \\
14,3 \%\end{array}$ & $5,4 \%$ & $\begin{array}{l}(0) \\
0 \%\end{array}$ & $\begin{array}{l}(0) \\
0 \%\end{array}$ & $93,3 \%$ \\
\hline 28. & $\begin{array}{l}\text { Doing work exceeds what is } \\
\text { assigned from your boss, } \\
\text { because I am not sure my } \\
\text { team will do the job properly }\end{array}$ & $\begin{array}{c}(60) \\
65,2 \%\end{array}$ & $\begin{array}{c}(8) \\
8,7 \%\end{array}$ & $\begin{array}{c}(12) \\
13,0 \%\end{array}$ & $\begin{array}{c}(12) \\
13,0 \%\end{array}$ & $\begin{array}{l}(0) \\
0 \%\end{array}$ & $76,7 \%$ \\
\hline 29. & $\begin{array}{l}\text { In carrying out tasks based } \\
\text { on what is believed to be } \\
\text { true, regardless of the advice } \\
\text { of friends }\end{array}$ & $\begin{array}{c}(30) \\
36,6 \%\end{array}$ & $\begin{array}{c}(24) \\
29,3 \%\end{array}$ & $\begin{array}{c}(12) \\
14,6 \%\end{array}$ & $\begin{array}{c}(16) \\
19,5 \%\end{array}$ & $\begin{array}{l}(0) \\
0 \%\end{array}$ & $68,3 \%$ \\
\hline 30. & $\begin{array}{l}\text { In carrying out the task of } \\
\text { imitating a friend that I think } \\
\text { is good }\end{array}$ & $\begin{array}{c}(80) \\
74,1 \%\end{array}$ & $\begin{array}{c}(16) \\
14,8 \%\end{array}$ & $\begin{array}{c}(12) \\
11,1 \%\end{array}$ & $\begin{array}{l}(0) \\
0 \%\end{array}$ & $\begin{array}{l}(0) \\
0 \%\end{array}$ & $90,0 \%$ \\
\hline 31. & $\begin{array}{l}\text { For the sake of the maximum } \\
\text { quality of work, in carrying } \\
\text { out the task is guided by } \\
\text { what is believed to be true, } \\
\text { by paying attention to } \\
\text { criticism and suggestions }\end{array}$ & $\left|\begin{array}{l}(100) \\
86,2 \%\end{array}\right|$ & $\begin{array}{c}(16) \\
13,8 \%\end{array}$ & $0,0 \%$ & $\begin{array}{l}(0) \\
0 \%\end{array}$ & $\begin{array}{l}(0) \\
0 \%\end{array}$ & $96,7 \%$ \\
\hline 32. & $\begin{array}{l}\text { In making a decision will } \\
\text { pay attention to the available } \\
\text { data }\end{array}$ & $\begin{array}{c}(80) \\
74,1 \%\end{array}$ & $\begin{array}{l}(16) \\
14,8 \%\end{array}$ & $\begin{array}{c}(12) \\
11,1 \%\end{array}$ & $\begin{array}{l}(0) \\
0 \%\end{array}$ & $\begin{array}{l}(0) \\
0 \%\end{array}$ & $90,0 \%$ \\
\hline 33. & $\begin{array}{l}\text { In solving problems faced, } \\
\text { be careful and pay attention } \\
\text { to friends' suggestions }\end{array}$ & $\begin{array}{c}(60) \\
57,7 \%\end{array}$ & $\begin{array}{c}(32) \\
30,8 \%\end{array}$ & $\begin{array}{c}(12) \\
11,5 \%\end{array}$ & $\begin{array}{l}(0) \\
0 \%\end{array}$ & $\begin{array}{l}(0) \\
0 \%\end{array}$ & $86,7 \%$ \\
\hline 34. & $\begin{array}{l}\text { For the sake of compactness } \\
\text { a team must be able to teach } \\
\text { the competencies I have with } \\
\text { friends }\end{array}$ & $\begin{array}{c}(70) \\
66,0 \%\end{array}$ & $224)$ & $\begin{array}{c}(12) \\
11,3 \%\end{array}$ & $\begin{array}{l}(0) \\
0 \%\end{array}$ & $\begin{array}{l}(0) \\
0 \%\end{array}$ & $88,3 \%$ \\
\hline 35. & $\begin{array}{l}\text { Can provide corrections and } \\
\text { answer friends' questions } \\
\text { about their lack of } \\
\text { understanding in carrying }\end{array}$ & $\begin{array}{c}(50) \\
51,0 \%\end{array}$ & $\begin{array}{c}(24) \\
24,5 \%\end{array}$ & $\begin{array}{c}(24) \\
24,5 \%\end{array}$ & $\begin{array}{l}(0) \\
0 \%\end{array}$ & $\begin{array}{l}(0) \\
0 \%\end{array}$ & $81,7 \%$ \\
\hline
\end{tabular}

\begin{tabular}{|l|l|l|l|l|l|l|l|}
\hline & out their tasks & & & & & & \\
\hline 36. & $\begin{array}{l}\text { An employee must be able to } \\
\text { prepare a work plan well }\end{array}$ & $\begin{array}{l}(120) \\
100 \%\end{array}$ & $\begin{array}{c}(0) \\
0 \%\end{array}$ & $\begin{array}{c}(0) \\
0 \%\end{array}$ & $\begin{array}{c}(0) \\
0 \%\end{array}$ & $\begin{array}{c}(0) \\
0 \%\end{array}$ & $100,0 \%$ \\
\hline 37. & $\begin{array}{l}\text { An employee must have a } \\
\text { good work ethic, meaning } \\
\text { that every day works }\end{array}$ & $\begin{array}{c}(90) \\
80,4 \%\end{array}$ & $\begin{array}{l}(16) \\
14 \%\end{array}$ & $\begin{array}{c}(6) \\
5,4 \%\end{array}$ & $\begin{array}{l}(0) \\
0 \%\end{array}$ & $\begin{array}{l}(0) \\
0 \%\end{array}$ & $93,3 \%$ \\
\hline
\end{tabular}
according to the target set

38. It is very necessary to understand technology and (40) (40) (18) (0) (0) $81,7 \%$ English for the sake of the $40,8 \% \quad 40,8 \% \quad 18,4 \% \quad 0 \% \quad 0 \%$ task

39. If you are assigned to take part in the education and \begin{tabular}{ll|l|l|l|l|l|} 
training program, it will (20) (64) (6) & (0) & (0) & (6) $3 \%$ & (0)
\end{tabular} \begin{tabular}{ll|l|l|l|l|l|} 
carry out well, even though & $21,3 \%$ & $68,1 \%$ & $6,4 \%$ & $4,3 \%$ & $0 \%$ & $78,3 \%$
\end{tabular} the training material is not desirable

40. Feeling that the competence possessed is not in (0) (0) (0) (56) (50) \begin{tabular}{ll|l|l|l|l|l|l}
$\begin{array}{l}\text { accordance with the } \\
\text { demands of the work that is }\end{array}$ & $0 \%$ & $0 \%$ & $0 \%$ & $52,8 \%$ & $47,2 \%$ & $88,3 \%$
\end{tabular} the task

\begin{tabular}{|l|l|l|l|l|l|l|l|}
\hline 41. & A professional must have the & $(40)$ & $(40)$ & $(18)$ & $0,0 \%$ & $0,0 \%$ & $81,7 \%$ \\
\hline
\end{tabular} skills needed for his job

42. Adjusting the skills that I \begin{tabular}{ll|llllll} 
have with the type of task & $(20)$ & $(64)$ & $(12)$ & $0,0 \%$ & $0,0 \%$ & $80,0 \%$
\end{tabular} assigned

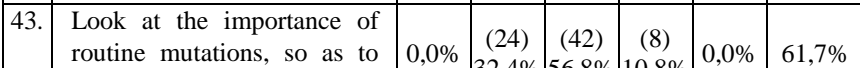
routine mutations, so as to $0,0 \% \quad 32,4 \% 56,8 \% 10,8 \%$

44. Believing that the experience \begin{tabular}{l|l|l|l|l|l|l|l} 
of various assignments will & $(20)$ & $(60)$ & $(15)$ & $(0)$ & $(0)$ & $79,2 \%$
\end{tabular} build intelligence

45. Every employee must have $\begin{array}{lllllllll}\text { an orientation that will be the } & (30) & (56) & (12) & (0) & (0) & 81,7 \%\end{array}$

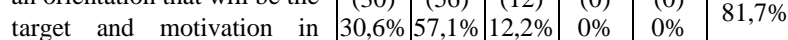
working

46. In carrying out the task an employee must have an orientation that will be used as an indicator of the quality of work to be carried out

47. Work orientation does not \begin{tabular}{ll|llllll} 
have to be theoretical, but & (85) & (20) & (6) & (0) & (0) & $92,5 \%$
\end{tabular}

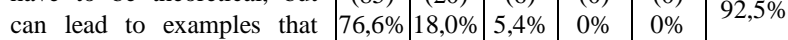
will be modeled

48. Believe in having the ability to carry out tasks in (30) (40) (12) (8) accordance with the tasks $33,3 \%$ 44,4\% $13,3 \% \quad 8,9 \% \quad 0 \% \quad 75,0 \%$ and functions

49. Will reject assignments from \begin{tabular}{lll|lll|l|l} 
superiors, if not in & $(0)$ & $(40)$ & $(24)$ & $(12)$ & $(0)$ & $63,3 \%$
\end{tabular}

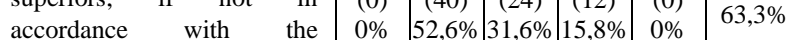
competencies they have \begin{tabular}{|l|l|l|l|l|l|l|l|}
\hline 50. & $\begin{array}{l}\text { Believe in being able to } \\
\text { carry out assignments from }\end{array}$ & $11,4 \%$ & $54,5 \%$ & $34,1 \%$ & $(0)$ & $(0)$ & $73,3 \%$ \\
$0 \%$
\end{tabular} superiors well

\begin{tabular}{|l|l|l|l|l|l|l|l|l|}
\hline 51. & $\begin{array}{l}\text { Ready to learn again to } \\
\text { adjust to work demands }\end{array}$ & $30,0 \%$ & $64,0 \%$ & $6,0 \%$ & $\begin{array}{l}(0) \\
0 \%\end{array}$ & $\begin{array}{l}(0) \\
0 \%\end{array}$ & $83,3 \%$ \\
\hline
\end{tabular}

52. Not looking at the importance of adjusting to the demands of competence in accordance with the development of society

53. Ready to adjust competencies in accordance

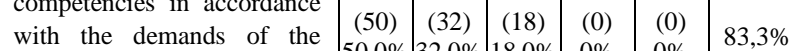
university's vision and mission (policy)

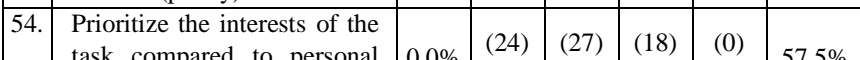
\begin{tabular}{cll|ccc|c|c} 
task compared to personal & $0,0 \%$ & $(24)$ & $(27)$ & $(18)$ & $(0)$ & $57,5 \%$ \\
\hline
\end{tabular} interests

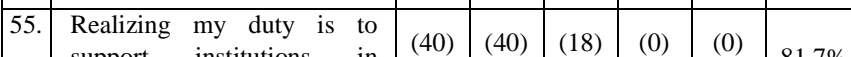

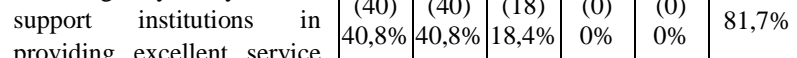




\begin{tabular}{|c|c|c|c|c|c|c|c|}
\hline & to the community & & & & & & \multirow[b]{2}{*}{$81,7 \%$} \\
\hline 56. & $\begin{array}{l}\text { If there is a family member } \\
\text { who is sick, then the } \\
\text { permission will be given to } \\
\text { my employer not to work }\end{array}$ & $\begin{array}{c}(40) \\
40,8 \%\end{array}$ & $\begin{array}{c}(40) \\
40,8 \%\end{array}$ & $\begin{array}{c}(18) \\
18,4 \%\end{array}$ & $\begin{array}{l}(0) \\
0 \%\end{array}$ & $\begin{array}{l}(0) \\
0 \%\end{array}$ & \\
\hline 57. & $\begin{array}{l}\text { Dare to be objective about } \\
\text { various things in the } \\
\text { environment }\end{array}$ & $\begin{array}{c}(30) \\
31,9 \%\end{array}$ & $\begin{array}{c}(40) \\
42,6 \%\end{array}$ & $\begin{array}{c}(24) \\
25,5 \%\end{array}$ & $\begin{array}{l}(0) \\
0 \%\end{array}$ & $\begin{array}{l}(0) \\
0 \%\end{array}$ & $78,3 \%$ \\
\hline 58. & $\begin{array}{l}\text { Supports mistakes } \text { or } \\
\text { programs carried out by } \\
\text { office friends who become } \\
\text { friends }\end{array}$ & $\begin{array}{l}(0) \\
0 \%\end{array}$ & $\begin{array}{l}(0) \\
0 \%\end{array}$ & $\begin{array}{l}(0) \\
0 \%\end{array}$ & $\begin{array}{c}(40) \\
36,4 \%\end{array}$ & $\begin{array}{c}(70) \\
63,6 \%\end{array}$ & $91,7 \%$ \\
\hline 59. & $\begin{array}{l}\text { Students become consumers } \\
\text { of Higher Education, so they } \\
\text { are given excellent service }\end{array}$ & $\begin{array}{c}(10) \\
11,1 \%\end{array}$ & $\begin{array}{c}(56) \\
62,2 \%\end{array}$ & $\begin{array}{c}(24) \\
26,7 \%\end{array}$ & $\begin{array}{l}(0) \\
0 \%\end{array}$ & $\begin{array}{l}(0) \\
0 \%\end{array}$ & $75,0 \%$ \\
\hline 60. & $\begin{array}{l}\text { Feel annoyed at students, } \\
\text { who when explained are } \\
\text { difficult to understand }\end{array}$ & $\begin{array}{l}(0) \\
0 \%\end{array}$ & $\begin{array}{l}(0) \\
0 \%\end{array}$ & $\begin{array}{c}(24) \\
25,5 \%\end{array}$ & $\begin{array}{c}(40) \\
42,6 \%\end{array}$ & $\begin{array}{c}(30) \\
31,9 \%\end{array}$ & $78,3 \%$ \\
\hline & \multicolumn{7}{|l|}{ Career development } \\
\hline 61. & 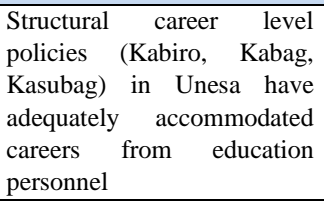 & $\begin{array}{l}(40) \\
40 \%\end{array}$ & $\begin{array}{l}(48) \\
48 \%\end{array}$ & $\begin{array}{l}(12) \\
12 \%\end{array}$ & $\begin{array}{l}(0) \\
0 \%\end{array}$ & $\begin{array}{l}(0) \\
0 \%\end{array}$ & $83,3 \%$ \\
\hline 62. & $\begin{array}{l}\text { Structural career level policy } \\
\text { (Kabiro, Kabag, sub-division } \\
\text { head) in Unesa is very } \\
\text { limited so it is unable to } \\
\text { accommodate careers from } \\
\text { education personnel }\end{array}$ & $\begin{array}{l}(0) \\
0 \%\end{array}$ & $\begin{array}{l}(0) \\
0 \%\end{array}$ & $\begin{array}{c}(12) \\
12,5 \%\end{array}$ & $\begin{array}{c}(64) \\
66,7 \%\end{array}$ & $\begin{array}{c}(20) \\
20,8 \%\end{array}$ & $80,0 \%$ \\
\hline 63. & $\begin{array}{l}\text { Considering that the } \\
\text { structural career is very } \\
\text { limited, it is considered } \\
\text { important that there is a } \\
\text { functional education policy } \\
\text { for educational personnel }\end{array}$ & $\begin{array}{c}(60) \\
58,3 \%\end{array}$ & $\begin{array}{c}(28) \\
27,2 \%\end{array}$ & $\begin{array}{c}(15) \\
14,6 \%\end{array}$ & $\begin{array}{l}(0) \\
0 \%\end{array}$ & $\begin{array}{l}(0) \\
0 \%\end{array}$ & $85,8 \%$ \\
\hline 64. & $\begin{array}{l}\text { Institutions need to establish } \\
\text { functional career policies } \\
\text { that are dynamic, so that they } \\
\text { can motivate and } \\
\text { accommodate potential } \\
\text { education personnel and do } \\
\text { not need to wait for limited } \\
\text { structural career vacancies. }\end{array}$ & $\begin{array}{c}(50) \\
50,0 \%\end{array}$ & $\begin{array}{c}(32) \\
32,0 \%\end{array}$ & $\begin{array}{c}(18) \\
18,0 \%\end{array}$ & $\begin{array}{l}(0) \\
0 \%\end{array}$ & $\begin{array}{l}(0) \\
0 \%\end{array}$ & $83,3 \%$ \\
\hline 65. & $\begin{array}{l}\text { Institutions need to provide } \\
\text { training to education } \\
\text { personnel who have just held } \\
\text { structural positions either } \\
\text { because of appointment or } \\
\text { transfer }\end{array}$ & $\begin{array}{c}(30) \\
32,6 \%\end{array}$ & $\begin{array}{c}(32) \\
34,8 \%\end{array}$ & $\begin{array}{c}(30) \\
32,6 \%\end{array}$ & $\begin{array}{l}(0) \\
0 \%\end{array}$ & $\begin{array}{l}(0) \\
0 \%\end{array}$ & $76,7 \%$ \\
\hline
\end{tabular}

From table 1 can be analyzed that the first integrity has urgency which is very important in supporting the success of one's performance to be of good quality, especially according to the head of the Subdivision at UNESA namely the details of the average results as follows: 1) indicators of the concept of integrity are very important, this is indicated by the average result that $75.8 \%$ said it was good if an employee had to have a concept of consistency between actions and values and principles. While $80 \%$ said it was good if someone had to have integrity that showed honesty, truth and action of someone. 2) on the commitment indicator shows that $71.7 \%$ stated that each employee must have an appointment to themselves or to others reflected in one's actions according to their duties and functions according to their position. While $75 \%$ expressed disapproval if each employee did not have to have a promise to themselves or their leaders, it was important to carry out their duties well. 3) responsibility indicators, $87.5 \%$ stated that even though the leadership was not in place, it would always carry out the duties in accordance with the main tasks that were owned because it was already a responsibility for the work. However, in carrying out their duties, $81.7 \%$ are still waiting for instructions from their superiors to comply with the wishes of the leadership. 4) indicators can be reliably stated with $90.8 \%$ of employees keeping all the promises promised to superiors, and $88.3 \%$ will keep all the promises promised to superiors even though they are at risk of being sanctioned. This is a trust and attitude of trust towards an integrity to work. 5) indicators of honesty with an average of $89.2 \%$ stating very honest, that is always saying in accordance with what is seen, heard and felt about various things that are around the working environment. Employees must always maintain harmony in the surrounding environment, so there is no need to say everything that is happening around us, to the average leader $96.7 \%$ strongly agree and on average $93.3 \%$ strongly agree to have high solidarity, so there is no need say the fault of the subordinate or friend to the leader. 6) the faithful indicator is an average of $99.2 \%$ stating that employees must have loyalty to their institution, by working hard, and on average $94.2 \%$ argue strongly agree that who is the boss will influence the performance donated to the institution. 7) the average commitment indicator strongly agrees if you will carry out the task without any sense of hesitation and turmoil that is $86.7 \%$, and on average $90 \%$ say that it will reprimand friends or subordinates, with great care and based on facts strong. 8) on the indicator of mastering and disciplining oneself an average of $96.7 \%$ said they would obey the prevailing regulations, without seeing the presence of superiors. On average $98.3 \%$ believe that self-discipline is the first step in building a career. On average $96.7 \%$ stated that the attitude of discipline is an important dimension rather than the closeness of the relationship with the leadership. 9) an average quality indicator of $96.7 \%$ states that working hard is something that can build self-quality, and an average of $90 \%$ states that they will not be angry with friends who have determined poor self-quality. Because this is a form of attention and motivation to be better.

Second. professionalism is a very important thing in doing work to provide good service, especially according to the head of the Subdivision at UNESA namely the details of the average results as follows: 1) professional concept indicators can be seen that $96.7 \%$ said a professional employee would carry out the task and work supported by abilities, knowledge, skills, ability to do and experience. However, on average $80 \%$ stated that they still adjust their capabilities with the demands of their main tasks. This still needs to be improved, and all of them feel embarrassed if they do not have the ability in accordance with their duties and functions, so they need to be very eager to learn to be better. 2) collaborative indicators can be seen that $93.3 \%$ have produced maximum work products, and must synergize with other friends. On average $71.7 \%$ in carrying out the task is still asking for help to other friends. However, on average $90 \%$ said they agreed and agreed to build mutual cohesiveness because it would be the main foundation for success. 3) indicators of mutual trust are stated on average 93.3\% carry out tasks that are charged properly every time there is a division of tasks from superiors. On average 
$76.7 \%$ always do their own work more than what is assigned from their superiors, because distrust of the team will be able to do the job well. 4) an open indicator of accepting the thoughts of others can be seen that on average $68.3 \%$ stated that every task was enough to be guided by what was believed to be true, regardless of the advice of friends. On average $90 \%$ said they would always imitate friends who were seen as good when carrying out tasks. On average $96.7 \%$ stated strongly agree that for the quality of the maximum work results, in carrying out the task will always be guided by what is believed to be true, by paying attention to criticism and suggestions. 5) indicators of looking for, seeing and solving problems that an average of $90 \%$ think that in making decisions always pay attention to existing data. On average $86.7 \%$ stated that they strongly agree when in solving the problems faced, then always be careful and pay attention to friends' suggestions. 6) the indicator of teaching competency, that is an average of $88.3 \%$, agrees that for the sake of cohesiveness of a team, it must be able to teach the competencies of friends. On average $81.7 \%$ said they agreed that they should be able to provide corrections and answer friends' questions about their lack of understanding in carrying out their duties. 7) indicators regulate plans that all strongly agree if an employee must be able to plan work well. On average $93.3 \%$ said they agreed that an employee must have a good work ethic, meaning that every day he worked in accordance with the targets set. 8) indicators of improving personal skills, namely an average of $81.7 \%$ strongly agree that it is very necessary to understand English technology and language for the sake of work. On average 78.3 agreed to be assigned to take part in the training and carry out well, even though the training material was not desirable. On average $88.3 \%$ stated that they felt strongly that the competencies they had were in accordance with the demands of the work they were assigned to.

Third, the characteristics of professionalism are the details of the average results as follows: 1) have high skills, that is an average of $81.7 \%$ strongly agree that a professional must have the skills needed for his job. On average $80 \%$ said they would always adjust their skills to the type of task assigned. 2) Having an average knowledge and experience and intelligence of $61.7 \%$ is still sufficient to consider the importance of routine mutations, so that they have diverse experiences. On average $79.2 \%$ said they were confident that the experience of various assignments would build their intelligence. 3) the indicator has an attitude oriented, that is an average of $81.7 \%$, it is very good if each employee must have an orientation that will be the target and motivation in working. However, in carrying out the task an average of $72.5 \%$ said that an employee must have an orientation will be used as an indicator of the quality of work to be carried out. On average $92.5 \%$ said it was very good if the work orientation did not have to be theoretical, but it could be directed to the example that would be used as an example. 4) Indicators have confidence in their own abilities, with an average of $75 \%$ stating that they are confident that they have the ability to carry out their duties according to their duties and functions. On average $63.3 \%$ will simply refuse assignments from superiors, if not in accordance with their competencies. On average $73.3 \%$ said they were confident they could carry out assignments from their superiors well, so this proved an optimistic attitude towards their own abilities without asking for help from others. 5) indicators of the ability to adapt to global phenomena refer to the vision and mission, which is an average of $83.3 \%$, are very ready to learn again to adjust to the demands of the work. On average $78 \%$ consider it important to adjust to the demands of competence in accordance with the development of society. On average $83.3 \%$ are always ready to adjust competencies in accordance with the demands of the university's vision and mission. 6) indicators of public interest averaging $57.5 \%$ less prioritizing the interests of the task than personal interests. On average $81.7 \%$ are very aware of their duty to support institutions in providing excellent service to the community. On average $81.7 \%$ said they agreed that if a family member was sick, then permission would be given to the employer not to work. 7) objective and independent indicators, with an average of $78.3 \%$ agreeing that they must have the courage to be objective about various things in the environment. On average $91.7 \%$ said they would always support mistakes or programs carried out by office friends who became friends. 8) indicators of service providers to the public, ie an average of $75 \%$ stated that students become consumers of Higher Education, therefore always providing excellent service. On average $78.3 \%$ stated that they always felt annoyed at students, who when explained were difficult to understand.

Fourth, structural development is a step towards further advancing and developing tasks in order to achieve good quality. As for the analysis, namely: 1) structural indicators averaged $83.3 \%$ stating that the structural career level policy (Kabiro, district head, sub-department head) in Unesa had adequately accommodated the career of the educational staff was good. On average $80 \%$ stated the structural career level policy (Kabiro, Kabag, Head of Subdivision) in Unesa was still quite limited so that it had not been able to accommodate the career of the teaching staff optimally. 2) an average functional indicator of $85.8 \%$ states that it is very important that there is a functional career policy for education personnel because the structural career is very limited. On average $83.3 \%$ said that institutions really need to establish functional career policies that are dynamic, so that they can motivate and accommodate potential education personnel and do not need to wait for limited structural career vacancies, 3) an internal education and training indicator that is an average of $76.7 \%$ stated strongly agree that the institution needs to provide training to education personnel who have just held structural positions either because of appointment or transfer.

\section{CONCLUSION}

This paper can be concluded that UNESA has been functionally good, but structurally it still needs improvement. Considering that the structural career is very limited, it is considered important that there is a functional career policy of education personnel, and institutions need to establish functional career policies that are dynamic, so as to motivate and accommodate potential education personnel and do not need to wait for limited structural career vacancies and need to provide debriefing of educational staff who have just held structural positions either due to appointment or transfer. Employee coaching is carried out 
with the intention of aiming at improving individual performance and organizational performance, so that organizational objectives can be achieved in accordance with the mission, as well as improving employee welfare through the results of the work done.

\section{ACKNOWLEDGMENT}

Thank to Directorate of Research and Community Service, Ministry of Research and Technology of Higher Education for supporting this study through The National Strategic Research Scheme Year 2017, the Work Order No. 1114/UN38/HK/LT/2017 on 28 dated August 2017.

\section{REFERENCES}

[1] National Science Board, "Preparing the Next Generation of STEM Innovators: Identifying and Developing our Nation's Human Capital," Chief Exec., 2010.

[2] A. M. Daud, J. Omar, P. Turiman, and K. Osman, "Creativity in Science Education," Procedia - Soc. Behav. Sci., 2012.

[3] R. Schendel and T. Mccowan, "Expanding higher education systems in low-and middle- income countries: the challenges of equity and quality," High. Educ., 2016.

[4] M. A. Peters and D. Araya, "Transforming American Education: Learning powered by technology," E-Learning and Digital Media, 2011.

[5] M. F. Giangreco, J. C. Suter, and S. M. Hurley, "Revisiting Personnel Utilization in Inclusion-Oriented Schools," J. Spec. Educ., 2013.

[6] H. Pashler, M. McDaniel, D. Rohrer, and R. Bjork, "Learning Styles: Concepts and Evidence," Psychol. Sci. Public Interes., 2008.

[7] J. W. Pellegrino, M. L. Hilton, and D. D. Learning, Education for life and work: developing transferable knowledge and skills in the 21st century. 2012.

[8] R. B. Fletcher, L. H. Meyer, H. Anderson, P. Johnston, and M. Rees, "Faculty and Students Conceptions of Assessment in Higher Education," High. Educ., 2012.

[9] J. C. Wayman, S. Midgley, and S. Stringfield, Leadership for databased decision making: Collaborative educator teams. 2006.

[10] C. K. Anand, V. Bisaillon, A. Webster, and B. Amor, "Integration of sustainable development in higher education - A regional initiative in Quebec (Canada)," J. Clean. Prod., 2015.

[11] F. Damanpour, "ORGANIZATIONAL INNOVATION: A METAANALYSIS OF EFFECTS OF DETERMINANTS AND MODERATORS.," Acad. Manag. J., 1991.
[12] B. Bana and W. McCourt, "Institutions and governance: Public staff management in Tanzania," Public Adm. Dev., 2006.

[13] T. S. Imaroh, "Entrepreneurship Education As a Strategy for Improving the Economical Independence and Competitive Ability of Society in Asean Economic Community (Aec) Era.," Int. J. Organ. Innov., 2016.

[14] P. Guramatunhu-Mudiwa and L. L. Bolt, "Does the gender of school personnel influence perceptions of leadership?," School Leadership and Management. 2012.

[15] H. C. Ngambi, "The relationship between leadership and employee morale in higher education," African J. Bus. Manag., 2011.

[16] T. Dyllick, "Responsible management education for a sustainable world," J. Manag. Dev., 2015.

[17] N. A. Owuor, "Higher Education in Kenya: The Rising Tension between Quantity and Quality in the Post-massification Period," High. Educ. Stud., 2012.

[18] S. Fine, "Practical guidelines for implementing preemployment integrity tests," Public Pers. Manage., 2013.

[19] E. Beddewela, C. Warin, F. Hesselden, and A. Coslet, "Embedding responsible management education - Staff, student and institutional perspectives," Int. J. Manag. Educ., 2017.

[20] G. Fitsimmons, "The foundational standard: Integrity," Bottom Line. 2008.

[21] J. Szekeres, "Professional staff carve out a new space," J. High. Educ. Policy Manag., 2011.

[22] J. W. Spain and M. M. Robles, "Academic integrity policy: The journey,” Bus. Commun. Q., 2011.

[23] L. E. Reybold, M. D. Halx, and A. L. Jimenez, "Professional Integrity in Higher Education: A Study of Administrative Staff Ethics in Student Affairs," J. Coll. Stud. Dev., 2008.

[24] M. Monga, "Integrity and its antecedent: A unified conceptual framework of integrity," J. Dev. Areas, 2016.

[25] A. Leigh, "Integrity: Are your leaders up to it?," Hum. Resour. Manag. Int. Dig., 2009.

[26] J. McCahill, O. Healy, S. Lydon, and D. Ramey, "Training Educational Staff in Functional Behavioral Assessment: A Systematic Review," Journal of Developmental and Physical Disabilities. 2014.

[27] C. Whitchurch, "Shifting identities and blurring boundaries: The emergence of third space professionals in UK higher education," High. Educ. Q., 2008.

[28] A. Etzioni, "Administrative and Professional Authority," ASHE Reader on Organization and Governance in Higher Education. 1964.

[29] I. McNay, "Values, Principles and Integrity: Academic and Professional Standards in UK Higher Education," High. Educ. Manag. Policy, 2007.

[30] T. McCowan, "Quality of higher education in Kenya: Addressing the conundrum," Int. J. Educ. Dev., 2018.

[31] T. McCowan, "Three dimensions of equity of access to higher education," Comp. A J. Comp. Int. Educ., 2016. 